\title{
Latency Constrained Simultaneous Wireless Information and Power Transfer
}

\author{
Dileep Kumar, Onel L. Alcaraz López, Satya Joshi and Antti Tölli \\ Centre for Wireless Communications, University of Oulu, Oulu, Finland \\ e-mail: \{dileep.kumar, onel.alcarazlopez, satya.joshi, antti.tolli\}@oulu.fi
}

\begin{abstract}
This paper studies a power splitting (PS)-based simultaneous wireless information and power transfer (SWIPT) multi-user system. Specifically, an optimization problem is formulated to minimize the average transmit power of the base station (BS) by jointly optimizing the transmit beamformer and receive $P S$ ratios, while meeting user-specific latency and energy harvesting (EH) requirements. We employ the Lyapunov optimization framework and provide a dynamic control algorithm for the time-average problem. The coupled and non-convex constraints are handled via the Successive Convex Approximation (SCA) technique, and a low-complexity iterative algorithm, where each step is computed in closed-form, is proposed by solving a system of Karush-Kuhn-Tucker (KKT) optimality conditions. The numerical results provide insights on the robustness of the proposed design to realize a power-efficient SWIPT system while ensuring latency and EH requirements in a dynamic network.
\end{abstract}

\section{INTRODUCTION}

Powering and supporting the seamless and autonomous operation of future Internet-of-Things (IoT) deployments is very challenging, given the exponential increase in the number of ubiquitous and heterogeneous IoT devices [1]. To this end, simultaneous wireless information and power transfer (SWIPT) technology becomes appealing as it allows full utilization of radio frequency (RF) resources via charging battery-limited/less wireless IoT devices over-the-air while conveying useful information [2]. Thus, it contributes to the realization of sustainable and green wireless IoT networks that scavenge the required power from ambient RF signals [1].

The downlink multi-antenna broadcast SWIPT system has attracted much attention from the research community, e.g., [3], [4]. Specifically, the setup includes a multi-antenna base-station (BS) communicating with several user equipments (UEs), where each UE may perform both energy harvesting (EH) and information decoding (ID) functions on the received signal, i.e., either by applying the time-switching (TS) [3] or power splitting (PS) [3], [4] protocol. Though a TS scheme simplifies the receiver design, it hinders the full exploitation of the SWIPT system, unlike PS schemes. A comprehensive overview of the aforementioned PS and TS schemes and other less popular SWIPT schemes, such as so-called the spatial switching and the integrated receiver are provided in [5].

The joint optimization of transmit beamformers and receive PS ratios has recently gained great interest [6]-[9]. The authors

This work was supported by the European Commission in the framework of the H2020-EUJ-02-2018 project under grant no. 815056 (5G-Enhance) and Academy of Finland under grants no. 318927 (6Genesis Flagship). in [6]-[9] minimized the BS transmit power in a downlink multi-user system with $\mathrm{EH}$ and a fixed quality-of-service (QoS) requirement. However, these schemes are studied for the static case (i.e., resource allocation for a given time instance). Furthermore, the SWIPT techniques in [6]-[9] were not originally designed to account for stringent latency requirements of, e.g., delay bounded critical applications for industrial automation scenarios, thus motivating the current work.

In this paper, we investigate the joint optimization of transmit beamformers and receive PS ratios that concurrently satisfy the user-specific latency and EH requirements of a PSbased SWIPT system. Specifically, we formulate a long-term time-average BS transmit power minimization problem subject to a minimum harvested power requirement and a maximum allowable queue backlog constraint for each user. To provide a tractable solution for the considered time-average problem, we provide a dynamic control algorithm using the Lyapunov optimization framework [10]. Furthermore, the coupled and non-convex constraints are handled via the Successive Convex Approximation (SCA) technique [11], [12], and a lowcomplexity algorithm, requiring the iterative evaluation of closed-form expressions, is proposed by solving a system of Karush-Kuhn-Tucker (KKT) conditions [13]. The simulation results manifest the robustness of the proposed design to realize a power-efficient SWIPT system for meeting userspecific latency and $\mathrm{EH}$ requirements in a dynamic network.

\section{SySTEM MODEL}

We consider downlink in a multi-user multiple-input singleoutput (MU-MISO) system with SWIPT, where all single antenna UEs are served by a BS with $N_{t}$ transmit antennas, as shown in Fig. 1. We define $\mathcal{K} \triangleq\{1,2, \ldots, K\}$ to represent the set of all UEs. For simplicity, but without loss of generality, we consider a time-slotted frame, where the slots are normalized to an integer value, e.g., $t \in\{1,2, \ldots\}$. Let $\mathbf{h}_{k}(t), \mathbf{f}_{k}(t) \in \mathbb{C}^{N_{t} \times 1}$ denote respectively the downlink channel vector and transmit beamformer during time slot $t$ for $k$-th UE. Then, the received signal can be expressed as

$$
y_{k}(t)=\mathbf{h}_{k}^{\mathrm{H}}(t) \mathbf{f}_{k}(t) d_{k}(t)+\sum_{u \in \mathcal{K} \backslash k} \mathbf{h}_{k}^{\mathrm{H}}(t) \mathbf{f}_{u}(t) d_{u}(t)+w_{k}(t),
$$

where $w_{k} \in \mathcal{C N}\left(0, \sigma_{k}^{2}\right)$ denotes the additive white Gaussian noise (AWGN), and $d_{k}$ is the downlink transmitted data symbol. Moreover, we assume independent and normalized data symbols, i.e., $\mathbb{E}\left\{d_{k} d_{u}^{*}\right\}=0$ and $\mathbb{E}\left\{\left|d_{k}\right|^{2}\right\}=1, \forall k, u \in \mathcal{K}$. 


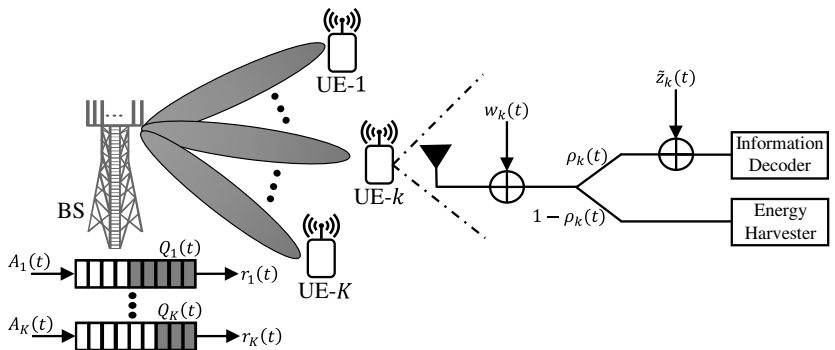

Fig. 1: SWIPT system with one BS and multiple UEs, where each UE employs PS to perform EH and ID simultaneously.

Similar to [3], [4], [6]-[9], we assume that each user $k$ implements PS on the received signal $y_{k}(t)$ for simultaneous ID and EH, as shown in Fig. 1. Let $\rho_{k}(t) \in[0,1]$ represent the PS ratio to the ID circuit of $k$-th UE during time slot $t$. Then, the portion of the signal split to the ID circuit can be expressed as

$$
\begin{aligned}
y_{k}^{\mathrm{ID}}(t)= & \sqrt{\rho_{k}(t)}\left(\mathbf{h}_{k}^{\mathrm{H}}(t) \mathbf{f}_{k}(t) d_{k}(t)\right. \\
& \left.+\sum_{u \in \mathcal{K} \backslash k} \mathbf{h}_{k}^{\mathrm{H}}(t) \mathbf{f}_{u}(t) d_{u}(t)+w_{k}(t)\right)+\tilde{z}_{k}(t),
\end{aligned}
$$

where $\tilde{z}_{k} \in \mathcal{C N}\left(0, \delta_{k}^{2}\right)$ is the additive noise at the ID circuit of $k$-th UE. The received signal-to-interference-plus-noise ratio (SINR) of $k$-th UE is given by

$$
\Gamma_{k}(t)=\frac{\rho_{k}(t)\left|\mathbf{h}_{k}^{\mathrm{H}}(t) \mathbf{f}_{k}(t)\right|^{2}}{\rho_{k}(t) \sum_{u \in \mathcal{K} \backslash k}\left|\mathbf{h}_{k}^{\mathrm{H}}(t) \mathbf{f}_{u}(t)\right|^{2}+\rho_{k}(t) \sigma_{k}^{2}+\delta_{k}^{2}} .
$$

On the other hand, the remaining portion of the signal split to the EH circuit of $k$-th UE during time slot $t$ is given by

$$
\begin{aligned}
y_{k}^{\mathrm{EH}}(t)=\sqrt{1-\rho_{k}(t)}\left(\mathbf{h}_{k}^{\mathrm{H}}(t) \mathbf{f}_{k}(t) d_{k}(t)\right. \\
\left.+\sum_{u \in \mathcal{K} \backslash k} \mathbf{h}_{k}^{\mathrm{H}}(t) \mathbf{f}_{u}(t) d_{u}(t)+w_{k}(t)\right) .
\end{aligned}
$$

Then, the harvested power at the EH of $k$-th UE is given by

$$
\mathrm{E}_{k}(t)=\zeta_{k}\left(1-\rho_{k}(t)\right)\left(\sum_{j \in \mathcal{K}}\left|\mathbf{h}_{k}^{\mathrm{H}}(t) \mathbf{f}_{j}(t)\right|^{2}+\sigma_{k}^{2}\right),
$$

where $\zeta_{k} \in(0,1]$ represents the energy conversion efficiency. Note that we are considering a linear model, as in [3], [4], [6]-[9], for analytical tractability and facilitate the discussions. However, the results and the performance trends shown in this paper should still approximately hold when considering more evolved EH models. In fact, this is a topic for future studies.

\section{A. Network Queueing \& Delay Model}

We assume that the BS has queue buffers to store the network layer data of UEs [10, Ch. 5]. Let $Q_{k}(t)$ denotes the queue backlog of $k$-th UE during time slot $t$, which evolves as

$$
Q_{k}(t+1)=\left[Q_{k}(t)-r_{k}(t)+A_{k}(t)\right]^{+}, \quad \forall k,
$$

where $[x]^{+} \triangleq \max (x, 0)$ and $r_{k}(t) \triangleq \log _{2}\left(1+\gamma_{k}(t)\right)$ is the achievable downlink rate of $k$-th UE. $A_{k}(t)$ denotes the data arrival with a mean arrival rate of $\mathbb{E}\left[A_{k}(t)\right]=\alpha_{k}, \forall k \in \mathcal{K}$.

According to Little's law, the average delay is proportional to long-term average queue length as $\lim _{T \rightarrow \infty} \frac{1}{T} \sum_{t=0}^{T-1} \mathbb{E}\left[Q_{k}(t)\right]$
[14, Ch. 1.4]. Thus, we use the queue backlogs $\left\{Q_{k}(t)\right\}$ as latency measure, and impose the allowable upper bound $\left\{Q_{k}^{\text {th }}\right\}$ for each time slot. Specifically, we consider a probabilistic constraint on user-specific queue length [11], defined as

$$
\operatorname{Pr}\left\{Q_{k}(t) \geq Q_{k}^{\text {th }}\right\} \leq \epsilon, \quad \forall t,
$$

and $\epsilon \ll 1$ is a tolerable probability for delay bound violation.

\section{B. Problem Formulation}

Our objective is to design a power-efficient resource allocation that satisfies both latency and EH requirements of UEs. Specifically, we jointly optimize transmit beamformers and receive PS ratios so as to minimize the BS average power subject to a probabilistic queue length constraint and a minimum harvested power requirement for each user. The network utility optimization problem can be formulated as

$$
\begin{aligned}
\min _{\mathbf{f}_{k}(t), \gamma_{k}(t), \rho_{k}(t), \forall t} & \lim _{T \rightarrow \infty} \frac{1}{T} \sum_{t=0}^{T-1}\left(\sum_{k \in \mathcal{K}} \mathbb{E}\left[\left\|\mathbf{f}_{k}(t)\right\|^{2}\right]\right) \\
\text { s.t. } & \operatorname{Pr}\left\{Q_{k}(t) \geq Q_{k}^{\text {th }}\right\} \leq \epsilon, \quad \forall k, \forall t, \\
& \gamma_{k}(t) \leq \Gamma_{k}(t), \quad \forall k, \forall t, \\
& \mathrm{e}_{k}(t) \leq \mathrm{E}_{k}(t), \quad \forall k, \forall t \\
& 0 \leq \rho_{k}(t) \leq 1, \quad \forall k, \forall t
\end{aligned}
$$

where $\mathbb{E}[\cdot]$ is with respect to data arrivals and random channel states. $\mathrm{e}_{k}(t)$ denotes a minimum harvested power requirement of $k$-th UE to support its receiver operations at each time slot.

\section{Optimal BeAmformer AND PS RATIOS DESIGN}

Problem (8) consists of a time-average sum-power objective function (8a), a non-linear probabilistic constraint (8b), coupled optimization variables $\left\{\mathbf{f}_{k}(t), \rho_{k}(t)\right\}_{\forall k}$, and non-convex expressions (8c), (8d), which cannot be addressed tractably. In this section, we elaborate on finding a solution for (8). First, we provide a dynamic control algorithm, specifically the driftplus-penalty function, using the Lyapunov optimization [10]. Next, the coupled and non-convex constraints are handled via SCA technique [11], [12], and an iterative algorithm, where each step is computed in closed-form expressions, is proposed by solving a system of KKT optimality conditions [13].

\section{A. Dynamic Control Algorithm via Lyapunov Framework}

We start by applying the well-known Markov's inequality [14], and linearize the probabilistic queue-length constraint as $\operatorname{Pr}\left\{Q_{k}(t) \geq Q_{k}^{\text {th }}\right\} \leq \mathbb{E}\left[Q_{k}(t)\right] / Q_{k}^{\text {th }} \leq \epsilon$, for all $k \in \mathcal{K}$. Thereby, problem (8) can be equivalently written as

$$
\begin{aligned}
\min _{\mathbf{f}_{k}(t), \gamma_{k}(t), \rho_{k}(t), \forall t} & \lim _{T \rightarrow \infty} \frac{1}{T} \sum_{t=0}^{T-1}\left(\sum_{k \in \mathcal{K}} \mathbb{E}\left[\left\|\mathbf{f}_{k}(t)\right\|^{2}\right]\right) \\
\text { s.t. } & \lim _{T \rightarrow \infty} \frac{1}{T} \sum_{t=0}^{T-1} \mathbb{E}\left[Q_{k}(t)\right] \leq \epsilon Q_{k}^{\text {th }}, \quad \forall k, \\
& \text { constraints }(8 \mathrm{c})-(8 \mathrm{e}) .
\end{aligned}
$$

Next, the time-average constraint (9b) is tackled by recasting it as a queue stability problem [10, Ch. 5]. Specifically, we define a virtual queue $Z_{k}(t)$ for each user $k$, which evolves as

$$
Z_{k}(t+1)=\left[Z_{k}(t)+Q_{k}(t+1)-\epsilon Q_{k}^{\text {th }}\right]^{+}, \quad \forall k .
$$


Note that the inequality constraint (9b) is strictly ensured only if the associated virtual queues $\left\{Z_{k}(t)\right\}_{\forall k}$ are stabilized [10, Theorem 2.5]. To stabilize the virtual queues, we now define a quadratic Lyapunov function $\mathcal{L}(\boldsymbol{\Psi}(t)) \triangleq \frac{1}{2} \sum_{k \in \mathcal{K}} Z_{k}(t)^{2}$, and its drift between two consecutive time slot as

$$
\begin{array}{r}
\triangle(\boldsymbol{\Psi}(t))=\frac{1}{2} \mathbb{E}\left[\sum_{k \in \mathcal{K}}\left(Z_{k}(t+1)^{2}-Z_{k}(t)^{2}\right) \mid \boldsymbol{\Psi}(t)\right], \\
\leq \Phi-\mathbb{E}\left[\sum_{k \in \mathcal{K}}\left(Q_{k}(t)+A_{k}(t)+Z_{k}(t)\right)\right. \\
\left.\quad \times \log _{2}\left(1+\gamma_{k}(t)\right) \mid \boldsymbol{\Psi}(t)\right],
\end{array}
$$

where $\boldsymbol{\Psi}(t)=\left(Z_{k}(t), Q_{k}(t) \mid \forall k \in \mathcal{K}\right)$ and $\Phi$ is a positive constant term which does not impact the system performance [10] (refer to [11, Section III] for further details on $\Phi$ ). We now define the a drift-plus-penalty function [10] for problem (9) as

$$
\triangle(\mathbf{\Psi}(t))+V \mathbb{E}\left[\sum_{k \in \mathcal{K}}\left\|\mathbf{f}_{k}(t)\right\|^{2} \mid \mathbf{\Psi}(t)\right],
$$

where $V \geq 0$ is a trade-off parameter. Hence, we can minimize the network utility, while ensuring the user-specific EH and latency performance, by minimizing the upper bound of driftplus-penalty function (12) subject to constraints (8c) - (8e). To do that, we use the principle of opportunistic minimization of an expectation [10, Ch. 1.8] and propose a dynamic control algorithm, which is outlined in Algorithm 1.

$$
\begin{aligned}
& \text { Algorithm 1: Dynamic algorithm for problem (8) } \\
& 1 \text { For a given time slot } t \text {, observe the queue backlog } \\
& \left\{Q_{k}(t)\right\} \text { and }\left\{Z_{k}(t)\right\} \text {, and solve the problem: }
\end{aligned}
$$

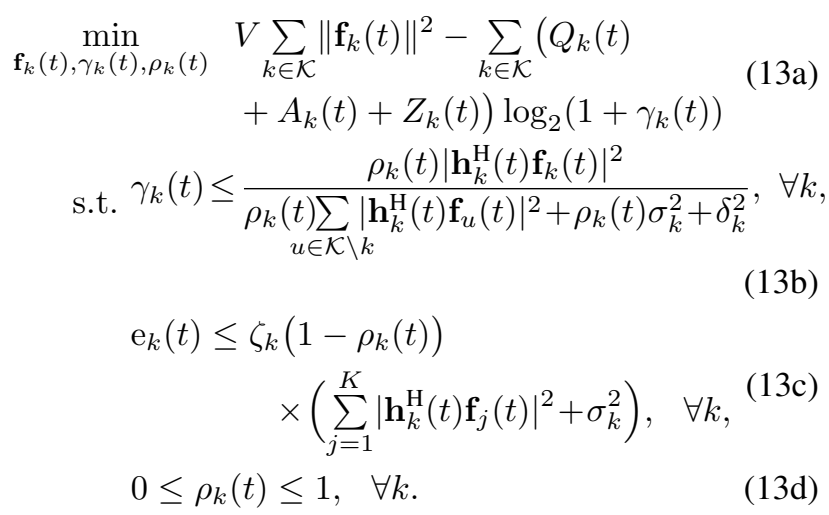

2 Update queues $Q_{k}(t+1)$ and $Z_{k}(t+1)$ by using (6) and (10), respectively, for all $k \in \mathcal{K}$

3 Set $t=t+1$, and go to step 1

\section{B. Solution via SCA framework}

The problem (13) is still intractable due to the non-convex SINR expression (13b), and the coupling between the optimization variables $\left\{\mathbf{f}_{k}(t), \rho_{k}(t)\right\}$ in constraint (13b) and (13c). Thus, we adopt the SCA framework [11], [12], wherein the non-convex problem (13) is recast as a sequence of convex subproblems, and then iteratively solved until convergence. In the following, time index $t$ is omitted for notation brevity.
1) Convex approximation for constraint (13b)

We start by rewriting SINR constraint (13b) as

$$
\gamma_{k} \leq \frac{\left|\mathbf{h}_{k}^{\mathrm{H}} \mathbf{f}_{k}\right|^{2}}{\sum_{u \in \mathcal{K} \backslash k}\left|\mathbf{h}_{k}^{\mathrm{H}} \mathbf{f}_{u}\right|^{2}+\sigma_{k}^{2}+\frac{\delta_{k}^{2}}{\rho_{k}}}, \quad \forall k .
$$

For compact representation, we define new functions as

$$
\begin{aligned}
& G_{k}\left(\mathbf{f}_{k}, \gamma_{k}\right) \triangleq \frac{\left|\mathbf{h}_{k}^{\mathrm{H}} \mathbf{f}_{k}\right|^{2}}{\gamma_{k}}, \\
& I_{k}\left(\mathbf{F}, \rho_{k}\right) \triangleq \sum_{u \in \mathcal{K} \backslash k}\left|\mathbf{h}_{k}^{\mathrm{H}} \mathbf{f}_{u}\right|^{2}+\sigma_{k}^{2}+\frac{\delta_{k}^{2}}{\rho_{k}} .
\end{aligned}
$$

where $\mathbf{F} \triangleq\left[\mathbf{f}_{1}, \mathbf{f}_{2}, \ldots, \mathbf{f}_{K}\right]$. Hence, expression (14) can be equivalently rewritten as

$$
I_{k}\left(\mathbf{F}, \rho_{k}\right)-G_{k}\left(\mathbf{f}_{k}, \gamma_{k}\right) \leq 0, \quad \forall k .
$$

Note that (15a) is a quadratic-over-linear function, and (15b) is a convex function with respect to the optimization variables. Hence, the left-hand side (LHS) of (16) is a difference of convex functions [13, Ch. 3]. Thus, the best convex approximation for constraint (16) can be obtained by replacing the function $G_{k}\left(\mathbf{f}_{k}, \gamma_{k}\right)$ with its first-order linear Taylor approximation around a fixed operating point $\left\{\mathbf{f}_{k}^{(i)}, \gamma_{k}^{(i)}\right\}$ as

$$
\begin{aligned}
\widetilde{G}_{k}\left(\mathbf{f}_{k}, \gamma_{k}\right) \triangleq 2 \Re\left\{\frac{\mathbf{f}_{k}^{(i) \mathrm{H}} \mathbf{h}_{k} \mathbf{h}_{k}^{\mathrm{H}}}{\gamma_{k}^{(i)}}\left(\mathbf{f}_{k}-\mathbf{f}_{k}^{(i)}\right)\right\} \\
+\frac{\left|\mathbf{h}_{k}^{\mathrm{H}} \mathbf{f}_{k}^{(i)}\right|^{2}}{\gamma_{k}^{(i)}}\left(1-\frac{\gamma_{k}-\gamma_{k}^{(i)}}{\gamma_{k}^{(i)}}\right) .
\end{aligned}
$$

2) Convex approximation for constraint (13c)

To begin with, we rewrite expression (13c) as

$$
\frac{\mathrm{e}_{k}}{\zeta_{k}\left(1-\rho_{k}\right)} \leq \sum_{j=1}^{K}\left|\mathbf{h}_{k}^{\mathrm{H}} \mathbf{f}_{j}\right|^{2}+\sigma_{k}^{2}, \quad \forall k .
$$

For compact representation, we define new functions as

$$
\begin{aligned}
& C_{k}\left(\rho_{k}\right) \triangleq \frac{\mathrm{e}_{k}}{\zeta_{k}\left(1-\rho_{k}\right)}, \\
& S_{k}(\mathbf{F}) \triangleq \sum_{j=1}^{K}\left|\mathbf{h}_{k}^{\mathrm{H}} \mathbf{f}_{j}\right|^{2}+\sigma_{k}^{2} .
\end{aligned}
$$

Hence, expression (18) can be equivalently rewritten as

$$
C_{k}\left(\rho_{k}\right)-S_{k}(\mathbf{F}) \leq 0, \quad \forall k .
$$

We can observe that both (19a) and (19b) are convex functions, and hence, the LHS of (20) is a difference of convex functions [13, Ch. 3]. Thus, to provide the best convex approximation for constraint (20), we replace the quadratic function $S_{k}(\mathbf{F})$ with its first-order linear Taylor approximation around a fixed operating point $\left\{\mathbf{f}_{k}^{(i)}\right\}$ as

$$
\widetilde{S}_{k}\left(\mathbf{f}_{k}\right) \triangleq 2 \sum_{j=1}^{K} \Re\left\{\mathbf{f}_{j}^{(i) \mathrm{H}} \mathbf{h}_{k} \mathbf{h}_{k}^{\mathrm{H}}\left(\mathbf{f}_{j}-\mathbf{f}_{j}^{(i)}\right)\right\}+\sum_{j=1}^{K}\left|\mathbf{h}_{k}^{\mathrm{H}} \mathbf{f}_{j}^{(i)}\right|^{2}+\sigma_{k}^{2} .
$$


Thereby, (13) can be approximated as the following convex subproblem:

$$
\begin{array}{rl}
\min _{\mathbf{f}_{k}, \gamma_{k}, \rho_{k}} & V \sum_{k \in \mathcal{K}}\left\|\mathbf{f}_{k}\right\|^{2}-\sum_{k \in \mathcal{K}}\left(Q_{k}+A_{k}+Z_{k}\right) \log _{2}\left(1+\gamma_{k}\right) \\
\text { s.t. } & \lambda_{k, 1}: I_{k}\left(\mathbf{F}, \rho_{k}\right)-\widetilde{G}_{k}\left(\mathbf{f}_{k}, \gamma_{k}\right) \leq 0, \quad \forall k, \\
& \lambda_{k, 2}: C_{k}\left(\rho_{k}\right)-\widetilde{S}_{k}(\mathbf{F}) \leq 0, \quad \forall k, \\
\lambda_{k, 3}: \rho_{k} \geq 0, \quad \forall k \\
\quad \lambda_{k, 4}: \rho_{k} \leq 1, \quad \forall k,
\end{array}
$$

where $\boldsymbol{\lambda}_{k}=\left[\lambda_{k, 1}, \lambda_{k, 2}, \lambda_{k, 3}, \lambda_{k, 4}\right]$ are Lagrange multipliers associated with each constraint. Note that the subproblem (22) provides an approximate solution in the proximity of a fixed operating point. Thus, by iteratively solving (22) and updating $\left\{\mathbf{f}_{k}^{(i)}, \gamma_{k}^{(i)}, \rho_{k}^{(i)}\right\}$ with the current SCA solution, as illustrated in Algorithm 2, we obtain the best local solution for (13).

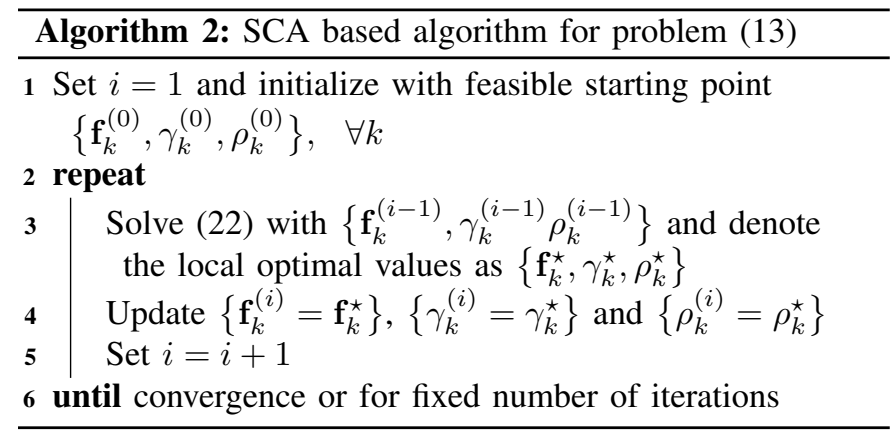

\section{Solution via KKT optimality conditions}

In this section, we provide an iterative algorithm by the method of Lagrange multipliers that does not rely on generic convex solvers. Specifically, we tackle (22) by iteratively solving a system of closed-form KKT optimality conditions [13, Ch. 5.5]. After some algebraic manipulation, we obtain the Lagrangian $\mathfrak{L}\left(\mathbf{F}, \gamma_{k}, \rho_{k}, \boldsymbol{\lambda}_{k}\right)$ of problem (22) as detailed in (23). Next, by differentiating (23) with respect to primal optimization variables $\left\{\mathbf{f}_{k}, \gamma_{k}, \rho_{k}\right\}$, we obtain the stationarity conditions for (22) as

$$
\begin{aligned}
& \nabla_{\mathbf{f}_{k}}: \mathbf{f}_{k}^{\mathrm{H}}\left(V \mathbb{I}+\sum_{u \in \mathcal{K} \backslash k} \lambda_{u, 1} \mathbf{h}_{u} \mathbf{h}_{u}^{\mathrm{H}}\right) \\
& =\lambda_{k, 1} \frac{\mathbf{f}_{k}^{(i) \mathrm{H}} \mathbf{h}_{k} \mathbf{h}_{k}^{\mathrm{H}}}{\gamma_{k}^{(i)}}+\mathbf{f}_{k}^{(i) \mathrm{H}} \sum_{j=1}^{K} \lambda_{j, 2} \mathbf{h}_{j} \mathbf{h}_{j}^{\mathrm{H}}, \\
& \nabla_{\gamma_{k}}: \frac{Q_{k}+A_{k}+Z_{k}}{1+\gamma_{k}}=\lambda_{k, 1} \frac{\left|\mathbf{h}_{k}^{\mathrm{H}} \mathbf{f}_{k}^{(i)}\right|^{2}}{\left(\gamma_{k}^{(i)}\right)^{2}}, \\
& \nabla_{\rho_{k}}: \lambda_{k, 1} \frac{\delta_{k}^{2}}{\rho_{k}^{2}}=\lambda_{k, 2} \frac{\mathrm{e}_{k}}{\zeta_{k}\left(1-\rho_{k}\right)^{2}}+\left(\lambda_{k, 4}-\lambda_{k, 3}\right) .
\end{aligned}
$$

Further, in addition to primal-dual feasibility, the KKT conditions include the complementary slackness, defined as

$$
\begin{aligned}
& \lambda_{k, 1}\left\{I_{k}\left(\mathbf{F}, \rho_{k}\right)-\widetilde{G}_{k}\left(\mathbf{f}_{k}, \gamma_{k}\right)\right\}=0, \quad \forall k, \\
& \lambda_{k, 2}\left\{C_{k}\left(\rho_{k}\right)-\widetilde{S}_{k}(\mathbf{F})\right\}=0, \quad \forall k, \\
& \lambda_{k, 3}\left\{0-\rho_{k}\right\}=0, \quad \forall k, \\
& \lambda_{k, 4}\left\{\rho_{k}-1\right\}=0, \quad \forall k .
\end{aligned}
$$

Let us assume Lagrange multipliers $\boldsymbol{\lambda}_{k}>0, \forall k$. Then, by using (24) and (25), and after the algebraic simplifications, the closed-form steps in the iterative method are

$$
\begin{aligned}
& \mathbf{f}_{k}^{(i) \mathrm{H}}=\left\{\lambda_{k, 1}^{(i-1)} \frac{\mathbf{f}_{k}^{(i-1) \mathrm{H}} \mathbf{h}_{k} \mathbf{h}_{k}^{\mathrm{H}}}{\gamma_{k}^{(i-1)}}+\mathbf{f}_{k}^{(i-1) \mathrm{H}} \sum_{j=1}^{K} \lambda_{j, 2}^{(i-1)} \mathbf{h}_{j} \mathbf{h}_{j}^{\mathrm{H}}\right\} \\
& \times\left(V \mathbb{I}+\sum_{u \in \mathcal{K} \backslash k} \lambda_{u, 1}^{(i-1)} \mathbf{h}_{u} \mathbf{h}_{u}^{\mathrm{H}}\right)^{-1}, \\
& \rho_{k}^{(i)}=1-\left\{\frac { \zeta _ { k } } { \mathrm { e } _ { k } } \left\{2 \sum_{j=1}^{K} \Re\left\{\mathbf{f}_{j}^{(i-1) \mathrm{H}} \mathbf{h}_{k} \mathbf{h}_{k}^{\mathrm{H}}\left(\mathbf{f}_{j}^{(i)}-\mathbf{f}_{j}^{(i-1)}\right)\right\}\right.\right. \\
& \left.\left.+\sum_{j=1}^{K}\left|\mathbf{h}_{k}^{\mathrm{H}} \mathbf{f}_{j}^{(i-1)}\right|^{2}+\sigma_{k}^{2}\right\}\right\}^{-1}, \\
& \gamma_{k}^{(i)}=2 \gamma_{k}^{(i-1)}+\left\{2 \Re\left\{\frac{\mathbf{f}_{k}^{(i-1) \mathrm{H}} \mathbf{h}_{k} \mathbf{h}_{k}^{\mathrm{H}}}{\gamma_{k}^{(i-1)}}\left(\mathbf{f}_{k}^{(i)}-\mathbf{f}_{k}^{(i-1)}\right)\right\}\right. \\
& \left.-\sum_{u \in \mathcal{K} \backslash k}\left|\mathbf{h}_{k}^{\mathrm{H}} \mathbf{f}_{u}^{(i)}\right|^{2}-\sigma_{k}^{2}-\frac{\delta_{k}^{2}}{\rho_{k}^{(i)}}\right\} \times \frac{\left(\gamma_{k}^{(i-1)}\right)^{2}}{\left|\mathbf{h}_{k}^{\mathrm{H}} \mathbf{f}_{k}^{(i-1)}\right|^{2}}, \\
& \lambda_{k, 1}^{(i)}=\frac{Q_{k}+A_{k}+Z_{k}}{1+\gamma_{k}^{(i)}} \times \frac{\left(\gamma_{k}^{(i-1)}\right)^{2}}{\left|\mathbf{h}_{k}^{\mathrm{H}} \mathbf{f}_{k}^{(i-1)}\right|^{2}}, \\
& \lambda_{k, 2}^{(i)}=\frac{\zeta_{k}\left(1-\rho_{k}^{(i-1)}\right)^{2}}{\mathrm{e}_{k}} \times\left\{\frac{\lambda_{k, 1}^{(i-1)} \delta_{k}^{2}}{\left(\rho_{k}^{(i-1)}\right)^{2}}-\lambda_{k, 4}^{(i-1)}+\lambda_{k, 3}^{(i-1)}\right\}, \\
& \lambda_{k, 3}^{(i)}=\left(\lambda_{k, 3}^{(i-1)}+\beta_{\lambda_{k, 3}}\left[0-\rho_{k}^{(i)}\right]\right)^{+}, \\
& \lambda_{k, 4}^{(i)}=\left(\lambda_{k, 4}^{(i-1)}+\beta_{\lambda_{k, 4}}\left[\rho_{k}^{(i)}-1\right]\right)^{+},
\end{aligned}
$$

where $\beta_{\lambda_{k, 3}}$ and $\beta_{\lambda_{k, 4}}$ are small positive step-sizes. Note that the Lagrange multipliers $\left\{\lambda_{k, 3}, \lambda_{k, 4}\right\}$ are iteratively updated based on violation of PS ratios (i.e., the complementary slackness condition) using the constrained sub-gradient method [13]. It is worth noting that due to the harvesting constraint, the beamformer design (26a) inherently has a multicast structure. The proposed method by iteratively solving a system of KKT equations is summarized in Algorithm 3.

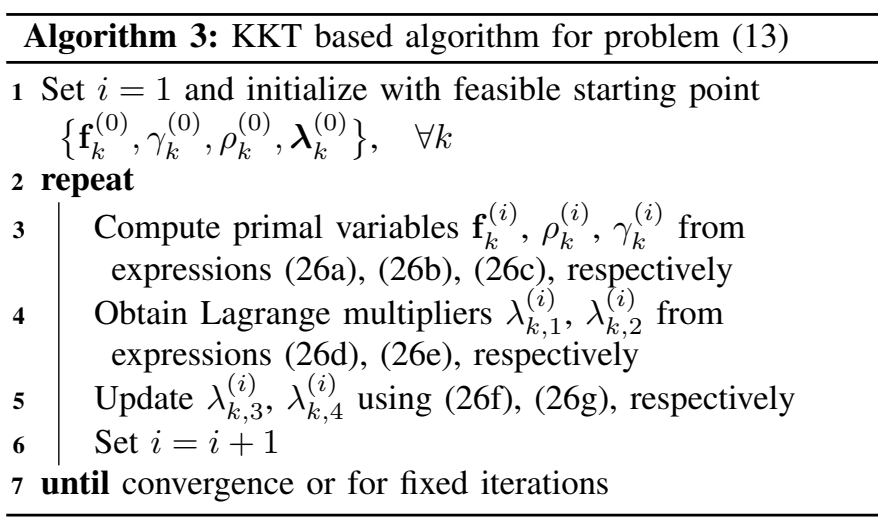

\section{Simulation Results}

We consider a SWIPT system with $K=4$ UEs being served by a BS equipped with a uniform linear array (ULA) of $N_{t}=8$ antennas. For simplicity, we assume the same parameters for all UEs, i.e., noise variance $\delta_{k}^{2}=-50 \mathrm{dBm}$ and $\sigma_{k}^{2}=-70 \mathrm{dBm}$; 


$$
\begin{aligned}
\mathfrak{L}\left(\mathbf{F}, \gamma_{k}, \rho_{k}, \boldsymbol{\lambda}_{k}\right)=\sum_{k \in \mathcal{K}}\left[V\left\|\mathbf{f}_{k}\right\|^{2}-\left(Q_{k}+A_{k}+Z_{k}\right) \log _{2}\left(1+\gamma_{k}\right)+\sum_{u \in \mathcal{K} \backslash k} \lambda_{u, 1}\left|\mathbf{h}_{u}^{\mathrm{H}} \mathbf{f}_{k}\right|^{2}\right. \\
+\lambda_{k, 1}\left\{\sigma_{k}^{2}+\frac{\delta_{k}^{2}}{\rho_{k}}-2 \Re\left\{\frac{\mathbf{f}_{k}^{(i) \mathrm{H}} \mathbf{h}_{k} \mathbf{h}_{k}^{\mathrm{H}}}{\gamma_{k}^{(i)}}\left(\mathbf{f}_{k}-\mathbf{f}_{k}^{(i)}\right)\right\}-2 \frac{\left|\mathbf{h}_{k}^{\mathrm{H}} \mathbf{f}_{k}^{(i)}\right|^{2}}{\gamma_{k}^{(i)}}+\gamma_{k} \frac{\left|\mathbf{h}_{k}^{\mathrm{H}} \mathbf{f}_{k}^{(i)}\right|^{2}}{\left(\gamma_{k}^{(i)}\right)^{2}}\right\}+\lambda_{k, 2}\left\{\left(\frac{1}{1-\rho_{k}}\right) \frac{\mathrm{e}_{k}}{\zeta_{k}}\right\} \\
\left.\quad-2 \sum_{j=1}^{K} \lambda_{j, 2} \Re\left\{\mathbf{f}_{k}^{(i) \mathrm{H}} \mathbf{h}_{j} \mathbf{h}_{j}^{\mathrm{H}}\left(\mathbf{f}_{k}-\mathbf{f}_{k}^{(i)}\right)\right\}-\lambda_{k, 2}\left\{\sum_{j=1}^{K}\left|\mathbf{h}_{k}^{\mathrm{H}} \mathbf{f}_{j}^{(i)}\right|^{2}+\sigma_{k}^{2}\right\}+\rho_{k}\left\{\lambda_{k, 4}-\lambda_{k, 3}\right\}-\lambda_{k, 4}\right] .
\end{aligned}
$$

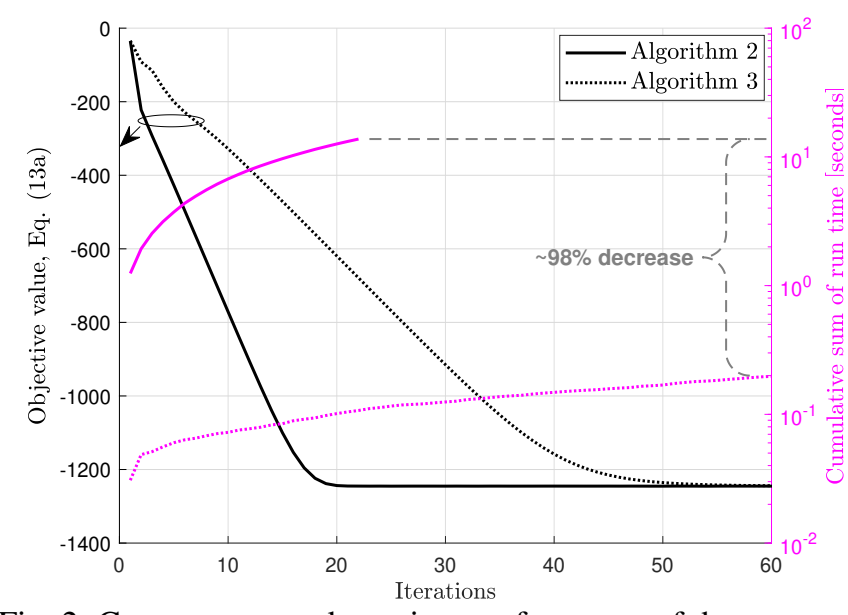

Fig. 2: Convergence and run time performance of the proposed algorithms with parameter $V=5$ and mean arrival $\alpha=15$ bits.

conversion efficiency of $\mathrm{EH} \zeta_{k}=0.1$; minimum harvested power requirements $\mathrm{e}_{k}(t)=10 \mathrm{dBm}, \forall t, k$ [9]. Further, we consider a Poisson arrival process $A_{k} \sim \operatorname{Pois}(\alpha)$, allowable queue backlog upper bound $Q_{k}^{\text {th }}=5$ bits and tolerable violation probability $\epsilon=10 \%$ in problem (8) [11].

We consider uncorrelated Rician fading to model the radio channel [9]. Specifically, the channel $\mathbf{h}_{k} \in \mathbb{C}^{N_{t} \times 1}$ of $k$-th UE consist of a deterministic line-of-sight $(\operatorname{LoS})$ path $\mathbf{h}_{k}^{\mathrm{L}}$ and a spatially uncorrelated non-LoS (NLoS) path $\mathbf{h}_{k}^{\mathrm{NL}}$, such that

$$
\mathbf{h}_{k}(t)=\sqrt{\frac{\kappa}{1+\kappa}} \mathbf{h}_{k}^{\mathrm{L}}(t)+\sqrt{\frac{1}{1+\kappa}} \mathbf{h}_{k}^{\mathrm{NL}}(t), \quad \forall t, k,
$$

where $\kappa$ is the Rician factor. We set $\kappa=5 \mathrm{~dB}$ unless stated otherwise. Moreover, the NLoS component is modeled using Rayleigh fading with the path-loss of $-40 \mathrm{~dB}$, independently for each time slot. Meanwhile, the LoS component follows the standard far-field ULA model, i.e., $\mathbf{h}_{k}^{\mathrm{L}}(t)=$ $10^{-4}\left[1, e^{-\mathrm{j} \pi \sin \left(\theta_{k}(t)\right)}, \ldots, e^{-\mathrm{j}\left(N_{t}-1\right) \pi \sin \left(\theta_{k}(t)\right)}\right]^{\mathrm{T}}$, where the azimuth angles $\theta_{k}(t) \in[-\pi / 2, \pi / 2], \forall t, k$, are randomly generated. We set $\beta_{\lambda_{k, 3}}=\beta_{\lambda_{k, 4}}=0.001$ in (26f) and (26g).

First, in Fig. 2, we investigate the convergence and the run time behavior of the proposed iterative algorithms for a given random channel realization. Note that the solution of Algorithm 2 is obtained directly from a convex optimization toolbox, SeDuMi [15], and the solution of Algorithm 3 is based on iterative evaluation of closed-form KKT equations (26). We can observe from Fig. 2 that both algorithms converge to the same objective value within a fairly small number of iterations. However, the per-iteration computational

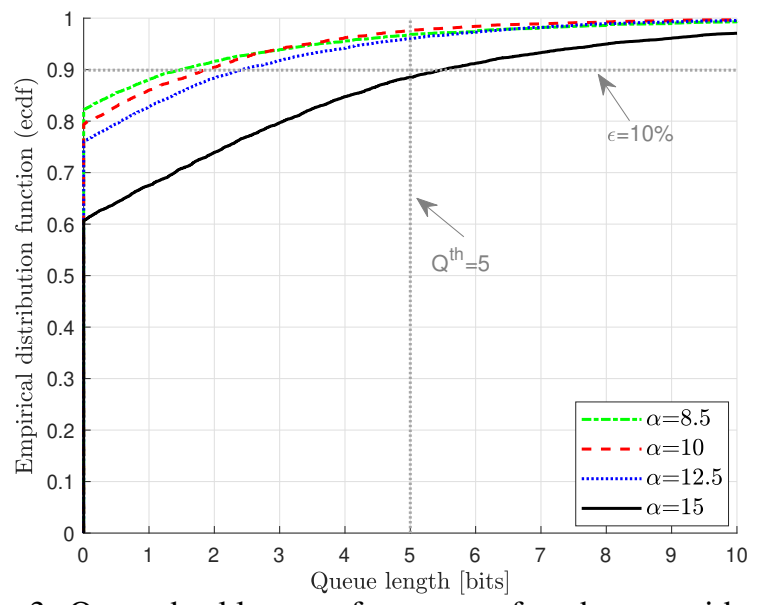

Fig. 3: Queue backlogs performance of each user with tradeoff parameter $V=5$.

complexity of Algorithm 2 scales with the length of the beamformer and requires $\mathcal{O}\left(N_{t}^{3.5}\right)$ arithmetic operations. On the contrary, the computational complexity of Algorithm 3 is mainly dominated by the inverse operation (26a), and each iteration requires $\mathcal{O}\left(N_{t}^{2.807}\right)$ arithmetic operations [13, Appendix C]. Furthermore, matrix inversion complexity in (26a) can be alleviate by computing the beamformer $\left\{\mathbf{f}_{k}\right\}_{\forall k}$ from the set of linear equations [13]. Thus, Algorithm 3 achieves comparable performance with a significant reduction in the run time and the computational complexity. Therefore, the KKT based iterative algorithm provides more practical, latencyconscious, and computationally efficient implementations for, e.g., the delay bounded factory automation scenarios.

In Fig. 3 and Fig. 4, we investigate the impact of different arrival $\alpha$ and trade-off parameter $V$ on system performance. Note that Fig. 3 is obtained with a fixed $V=5$. However, a similar performance can be observed for different values of $V$, which are not included due to space limitations. It can be concluded from Fig. 3 that irrespective of the mean arrival $\alpha$, the proposed method ensures the maximum queue backlogs of each user $k$ (i.e., $Q_{k}^{\text {th }}=5$ ) within tolerable violation probability $\epsilon=10 \%$. Furthermore, we can observe a similar behavior for the minimum harvested power requirements of each user, which is not included due to space limitations. Thus, the proposed method strictly satisfies the constraints (8b), (8d).

Next, we examine the average BS transmit power performance in Fig. 4. Result shows that the sum power decreases with the increase in the value of the trade-off parameter $V$. This is an expected behavior, e.g., $V$ can be anticipated as a 


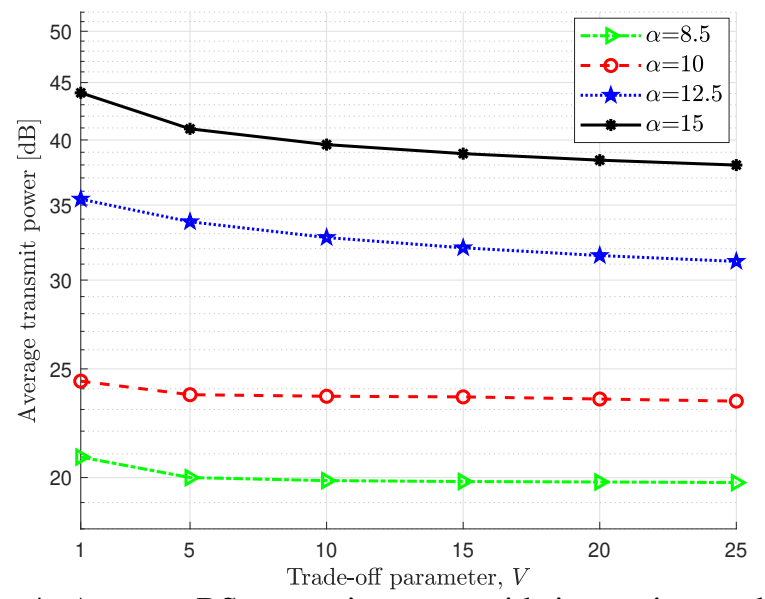

Fig. 4: Average BS transmit power with increasing trade-off parameter $V$ and different mean arrivals.

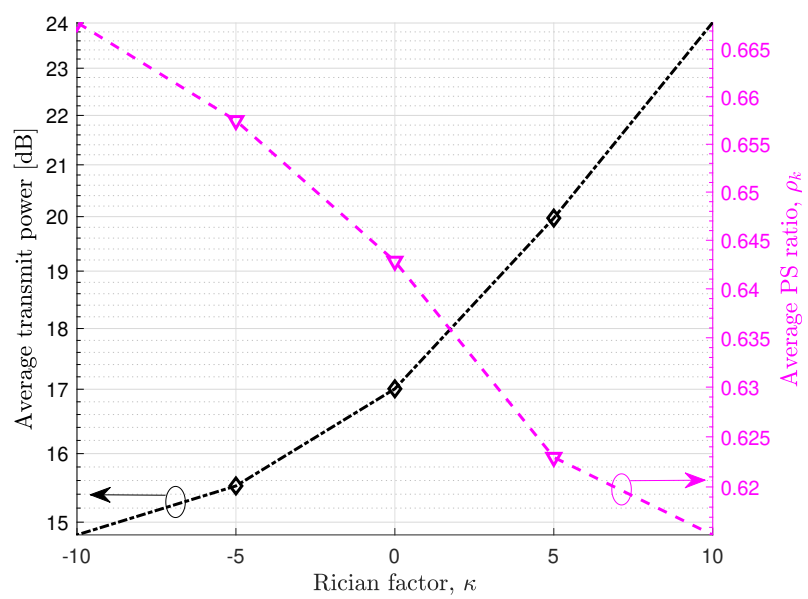

Fig. 5: Average PS ratio $\rho_{k}$ with increasing Rician factor $\kappa$, trade-off parameter $V=5$ and mean arrival $\alpha=8.5$ bits.

scaling factor (see (13a)). Thus, higher values of $V$ emphasize the minimization of transmit power over the queue length, until the queue backlogs become substantially larger than the sumpower objective value. Furthermore, the BS average transmit power significantly increases with the increase in mean arrival rate. This is mainly due to the raise in queue backlogs (6), which become more stringent. For that, the BS consumes relatively more power and attempt to increase the achievable rates in order to satisfy the queue backlog constraint (8b).

Fig. 5 shows that the average PS ratio $\rho_{k}$ decreases with the increase in the Rician factor $\kappa$ in (27). Thus, to ensure the minimum harvested power requirements (8d), a larger portion of the received signal $\left(1-\rho_{k}\right)$ is split into the EH circuit of each user. It is worth highlighting that the PS ratios to the EH circuit also increase as the minimum harvested power requirements become more stringent. Furthermore, with an increasing Rician factor $\kappa$, the BS tries to increase the downlink rate as much as possible, and thus, consumes relatively more power for ensuring the constraints (8b), (8d).

Therefore, even after the proposed relaxations to problem (8), the solution still allows to satisfy the desired user- specific latency and EH requirements, i.e., constraints (8b)(8d) are strictly met with the minimum transmit power. Thus, by suitable parameterization, one can easily achieve latency and EH requirements of, e.g., industrial-grade delay bounded critical applications for the factory automation scenarios.

\section{CONClusion}

In this paper, we considered a PS based SWIPT system and provided a joint optimization of transmit beamformers and receive PS ratios. Specifically, we considered a long-term timeaverage BS transmit power minimization problem concurrently satisfying the user-specific latency and EH requirements. To provide a tractable solution, we employed the Lyapunov optimization framework and provided a dynamic control algorithm for the time-average problem. Furthermore, the coupled and non-convex constraints were handled via the SCA technique, and a closed-form iterative algorithm was proposed by solving a system of KKT optimality conditions. The simulation results manifested the robustness of the proposed design to realize a power-efficient SWIPT system for industrial-grade delay bound applications for the factory automation scenarios.

\section{REFERENCES}

[1] O. L. A. López, H. Alves, R. D. Souza, S. Montejo-Sánchez, E. M. G. Fernández, and M. Latva-Aho, "Massive wireless energy transfer: Enabling sustainable IoT toward 6G era," IEEE Internet Things J., vol. 8, no. 11 , pp. 8816-8835, 2021.

[2] K. W. Choi, S. I. Hwang, A. A. Aziz, H. H. Jang, J. S. Kim, D. S. Kang, and D. I. Kim, "Simultaneous wireless information and power transfer (SWIPT) for Internet of Things: Novel receiver design and experimental validation," IEEE Internet Things J., vol. 7, no. 4, pp. 2996-3012, 2020.

[3] R. Zhang and C. K. Ho, "MIMO broadcasting for simultaneous wireless information and power transfer," IEEE Trans. Wireless Commun., vol. 12, no. 5, pp. 1989-2001, 2013.

[4] X. Zhou, R. Zhang, and C. K. Ho, "Wireless information and power transfer: Architecture design and rate-energy tradeoff," IEEE Trans. Commun., vol. 61, no. 11, pp. 4754-4767, 2013.

[5] X. Lu, P. Wang, D. Niyato, D. I. Kim, and Z. Han, "Wireless networks with RF energy harvesting: A contemporary survey," IEEE Commun. Surveys Tuts., vol. 17, no. 2, pp. 757-789, 2015.

[6] S. Gautam, E. Lagunas, S. Chatzinotas, and B. Ottersten, "Feasible point pursuit and successive convex approximation for transmit power minimization in SWIPT-multigroup multicasting systems," IEEE Trans. on Green Commun. Netw., pp. 1-1, 2021.

[7] Q. Shi, L. Liu, W. Xu, and R. Zhang, "Joint transmit beamforming and receive power splitting for MISO SWIPT systems," IEEE Trans. Wireless Commun., vol. 13, no. 6, pp. 3269-3280, 2014.

[8] M. R. A. Khandaker and K. Wong, "SWIPT in MISO multicasting systems," IEEE Wireless Commun. Lett., vol. 3, no. 3, 2014.

[9] Q. Shi, W. Xu, T. Chang, Y. Wang, and E. Song, "Joint beamforming and power splitting for MISO interference channel with SWIPT: An SOCP relaxation and decentralized algorithm," IEEE Trans. Signal Process., vol. 62, no. 23, pp. 6194-6208, 2014.

[10] M. J. Neely, Stochastic Network Optimization with Application to Communication and Queueing Systems, ser. Synthesis Lectures on Communication Networks. Morgan \& Claypool, 2010, vol. 7.

[11] D. Kumar, S. K. Joshi, and A. Tölli, "Latency-aware reliable mmwave communication via multi-point connectivity," in Proc. IEEE Global Commun. Conf., 2020, pp. 1-6.

[12] D. Kumar, J. Kaleva, and A. Tölli, "Blockage-aware reliable mmWave access via coordinated multi-point connectivity," IEEE Trans. Wireless Commun., vol. 20, no. 7, pp. 4238-4252, 2021.

[13] S. Boyd and L. Vandenberghe, Convex optimization. Cambridge university press, 2004.

[14] D. Gross, J. F. Shortle, J. M. Thompson, and C. M. Harris, Fundamentals of queueing theory. John Wiley \& Sons, 2008.

[15] M. Grant and S. Boyd, "CVX: Matlab software for disciplined convex programming, version 2.1," http://cvxr.com/cvx, Mar. 2014. 